\title{
EFECTO DE DIFERENTES DOSIS DE LODO DE LA CRIANZA DE SALMONES EN EL CULTIVO DE PAPA Y SU EFECTO RESIDUAL EN BALLICA ANUAL
}

\author{
Effect of different rates of cage salmon sludge on potato crop and its residual \\ effect on annual ryegrass
}

\author{
Nolberto Teuber K. ${ }^{1 *}$, Francisco Salazar S. ${ }^{1}$, Marta Alfaro V. ${ }^{1}$, y Aldo Valdebenito B. ${ }^{1}$
}

\section{A B S T R A C T}

Intensive salmon farming generates organic residues which have a potential use in agricultural soils. The objective of this study was to evaluate the effect of three salmon sludge rates on potato (Solanum tuberosum) crop development and its residual effect on annual ryegrass (Lolium multiflorum). The field experiment was carried out on an Andisoil from Osorno Serie (Hapludands), located in Osorno $\left(40^{\circ} 35^{\prime} \mathrm{S} ; 73^{\circ} 08^{\prime} \mathrm{W}\right)$, Chile. Treatment were three salmon sludge rates $\left(50,100,200 \mathrm{t} \mathrm{ha}^{-1}\right)$, a control (no fertilizer), and an inorganic fertilizer treatment. The salmon sludge was incorporated into the soil and then potato tubers $\mathrm{cv}$. Yagana-INIA were planted. After the harvest, an annual ryegrass was seeded. Total potato yield with inorganic fertilizer was $64.3 \mathrm{t} \mathrm{ha}^{-1}$, significantly superior to the results with the salmon sludge rates and the control $(\mathrm{P} \leq 0.05)$. There were no differences $(\mathrm{P}>0.05)$ among the fish sludge rates $(45.6$ to $\left.47.5 \mathrm{t} \mathrm{ha}^{-1}\right)$ and the control treatment $\left(39.4 \mathrm{t} \mathrm{ha}^{-1}\right)$. In addition, there were no differences $(P>0.05)$ on tuber weight, but the number of tubers per plant was different $(P \leq 0.05)$. Annual ryegrass yield was significantly different $(\mathrm{P} \leq 0.05)$ between fertilizer treatments and the control. Salmon sludge did not affect potato or ryegrass yield or development and increased P Olsen and cation exchange capacity in the soil.

Key words: salmon sludge, volcanic soil, chemical fertilizer, Solanum tuberosum, Lolium multiflorum.

\section{R E S U M E N}

La producción intensiva de salmones genera residuos orgánicos que tienen un uso potencial en suelos agrícolas. El objetivo del presente estudio fue evaluar el efecto de tres dosis de lodo de salmón en el desarrollo del cultivo de papa (Solanum tuberosum) y su efecto residual en ballica anual (Lolium multiflorum). El ensayo se estableció en un suelo Andisol de la serie Osorno (Typic Hapludands), ubicado en Osorno $\left(40^{\circ} 35^{\prime} \mathrm{S} ; 7^{\circ} 08^{\prime} \mathrm{O}\right)$, Chile. Los tratamientos fueron tres dosis de lodo $\left(50,100\right.$ y $\left.200 \mathrm{t} \mathrm{ha}^{-1}\right)$, un control (sin fertilización) y un tratamiento de fertilización inorgánica. El lodo se incorporó al suelo, posteriormente se plantó papa cv. Yagana-INIA y después de la cosecha se sembró ballica anual cv. Sabalan. El rendimiento de papa con fertilización inorgánica fue 64,3 $\mathrm{t} \mathrm{ha} \mathrm{a}^{-1}$, superior a los resultados obtenidos con las dosis de lodo y el control $(\mathrm{P} \leq 0,05)$. No hubo diferencias estadísticas $(\mathrm{P}>0,05)$ entre las distintas dosis de lodo evaluadas $\left(45,6\right.$ a $\left.47,5 \mathrm{t} \mathrm{ha}^{-1}\right)$ y tampoco con el control $\left(39,4 \mathrm{t} \mathrm{ha}^{-1}\right)$. No se observaron diferencias $(\mathrm{P}>0,05)$ en el peso por tubérculo, pero el número de tubérculos por planta fue diferente $(\mathrm{P} \leq 0,05)$. El rendimiento de ballica anual fue significativamente diferente $(\mathrm{P} \leq 0,05)$ en los tratamientos fertilizados comparados con el control. El lodo no afectó la emergencia ni el desarrollo de los cultivos, pero incrementó el contenido de P Olsen y la suma de bases de intercambio en el suelo.

Palabras clave: lodo salmones, suelo volcánico, fertilización inorgánica, papa, Solanum tuberosum, Lolium multiflorum.

\footnotetext{
${ }^{1}$ Instituto de Investigaciones Agropecuarias, Centro Regional de Investigación Remehue, Km 8 Ruta 5 Norte, Casilla 24-O, Osorno, Chile. E-mail: nteuber@inia.cl *Autor para correspondencia.

Recibido: 14 de junio de 2006. Aceptado: 18 de agosto de 2006.
} 


\section{INTRODUCCIÓN}

En el año 2005 la exportación chilena de truchas y salmones superó las 384.000 toneladas, equivalente a US\$1.720 millones; se estima que para el año 2010 en Chile se producirán alrededor de US $\$ 3.000$ millones de este producto (Salmón Chile, 2006).

En la producción de cada tonelada de salmón se generan 1,4 toneladas de desechos orgánicos, provenientes tanto de las jaulas de crianza como del proceso de faenamiento (Rodríguez, 1993), producto que contiene diferentes nutrientes que podrían utilizarse en sistemas agrícolas, reduciendo la contaminación del medio acuícola. Ackefors y Enell (1994) y Niklitschek et al. (2006), señalan que por cada tonelada de salmón cosechado, 35 a $78 \mathrm{~kg}$ de nitrógeno $(\mathrm{N})$ y 7 a $10 \mathrm{~kg}$ de fósforo $(\mathrm{P})$ son liberados al medio ambiente. El $\mathrm{N}$ se pierde principalmente en forma disuelta como amoníaco (62\%) y urea $(9 \%)$, quedando el resto en la fracción sólida de las fecas (29\%), que se acumulan bajo las balsas-jaulas. Estos desechos orgánicos se descomponen por la acción de los microorganismos, produciendo problemas de eutroficación y disminución del oxígeno en el cuerpo de agua, condiciones que afectan directamente la vida marina y lacustre (Stickney, 2002), incluyendo a los salmones, los que no pueden desarrollarse en un ambiente anóxico.

El aprovechamiento de diferentes residuos orgánicos (purines de lechería, desechos urbanos, riles industriales, lodos acuícolas, entre otros) ha aumentado exponencialmente en la última década, debido a su valor agronómico y a su capacidad para aumentar la calidad y la cantidad de materia orgánica, por su valor como enmienda y su posibilidad de incrementar el contenido de nutrientes en el suelo (Mazzarino et al., 1998; Laos et al., 2000), principalmente fósforo, calcio $(\mathrm{Ca})$ y magnesio $(\mathrm{Mg})$.

Estudios nacionales y extranjeros han aportado antecedentes para su uso potencial como fertilizante en praderas y cultivos (Smith, 1985; Mazzarino et al., 1997; Teuber et al., 2004; 2005). En general existe escasa información a nivel mundial de su uso en suelos agrícolas, y no se conoce información publicada de su utilización en el cultivo de papa. Estudios extranjeros y nacionales han caracterizado física y químicamente estos lodos (Westerman et al., 1993; Naylor et al., 1999; Salazar y Saldaña, 2007), señalando que existe un uso potencial para su incorporación al suelo debido a su aporte de macro y micro nutrientes.

El presente estudio tiene como objetivo evaluar el efecto de la incorporación de lodo de lago proveniente de la crianza intensiva de salmonídeos en el comportamiento productivo del cultivo de papa y su efecto residual en ballica anual.

\section{MATERIALES Y MÉTODOS}

El estudio se realizó entre el año 2002 y 2003 en un suelo trumao serie Osorno (Typic Hapludans) (CIREN, 2003), ubicado en el Centro Regional de Investigación Remehue, perteneciente al Instituto de Investigaciones Agropecuarias (INIA), Osorno

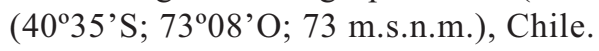

El lodo se colectó bajo balsas-jaulas de crianza de salmones ubicadas en el Lago Llanquihue, sector El Encanto $\left(40^{\circ} 47^{\prime} \mathrm{S} ; 7^{\circ} 28^{\prime} \mathrm{O}\right)$, provincia de Llanquihue, Región de Los Lagos. El lodo se extrajo a fines de septiembre de 2002 en el perfil de 8 a 10 m de profundidad bajo las jaulas, utilizando un equipo mecánico a control remoto para succionar y elevar el residuo a la superficie, almacenándolo en piscinas flotantes donde se floculó (proceso por el cual se concentró y se separó el agua de los sólidos). La fase líquida se devolvió al cuerpo de agua y el material restante se trasladó mediante una bomba, depositándose en contenedores plásticos para su posterior traslado al sitio experimental. Los contenedores se mantuvieron a temperatura ambiente y bajo sombra.

Al inicio del ensayo se realizó la caracterización química del lodo (Cuadro 1). La materia orgánica se determinó por calcinación de cenizas $\left(550^{\circ} \mathrm{C}\right.$ por 6 horas) en una mufla de acuerdo a la metodología descrita por AOAC (1984). La acidez (pH) se determinó por el método electrométrico revisado por Sadzawka (1990). Los macro nutrientes totales (P, $\mathrm{K}, \mathrm{Ca}, \mathrm{Na} \mathrm{y} \mathrm{Mg}$ ) y los micro nutrientes $(\mathrm{Zn}, \mathrm{Fe}$, $\mathrm{Mn}, \mathrm{Cu}$ y $\mathrm{Al}$ ) se determinaron por digestión de acuerdo a la metodología revisada por AOAC (1984) y Sadzawka (1990).

El P total fue determinado por colorimetría (Espectrofotómetro UV/VIS Perkin-Elmer modelo Lambda 3B, Norwalk, Connecticut, USA). El K y Na fueron determinados por espectrofotometría de emisión atómica (Perkin-Elmer modelo 1100B, 


\section{Cuadro 1. Análisis químico de lodo de salmones proveniente del Lago Llanquihue, incorporado en el cultivo de papa.}

Table 1. Chemical analysis of salmon sludge from Lake Llanquihue, incorporated into the potato crop.

\begin{tabular}{|c|c|c|}
\hline Parámetro & Conce & entración \\
\hline Materia seca, g $100 \mathrm{~g}^{-1}$ & 10,8 & 0,19 \\
\hline Cenizas, g $100 \mathrm{~g}^{-1}$ & 77,3 & 0,01 \\
\hline Materia orgánica, g $100 \mathrm{~g}^{-1}$ & 22,7 & 0,01 \\
\hline $\mathrm{pH}$ & 7,3 & 0,02 \\
\hline $\mathrm{N}$ total, g $100 \mathrm{~g}^{-1}$ peso fresco & 0,19 & 0,001 \\
\hline $\mathrm{N}-\mathrm{NH}_{4}$, g $100 \mathrm{~g}^{-1}$ peso fresco & 0,07 & 0,001 \\
\hline $\mathrm{C}, \mathrm{g} 100 \mathrm{~g}^{-1}$ & 12,6 & 0,01 \\
\hline Relación C:N & 7,2 & 0,07 \\
\hline$P, g 100 g^{-1}$ & 0,84 & 0,012 \\
\hline $\mathrm{Ca}, \mathrm{g} 100 \mathrm{~g}^{-1}$ & 2,7 & 0,05 \\
\hline $\mathrm{K}, \mathrm{g} 100 \mathrm{~g}^{-1}$ & 0,09 & 0,001 \\
\hline $\mathrm{Mg}, \mathrm{g} 100 \mathrm{~g}^{-1}$ & 0,37 & 0,009 \\
\hline $\mathrm{Na}, \mathrm{g} 100 \mathrm{~g}^{-1}$ & 0,16 & 0,009 \\
\hline $\mathrm{Zn}, \mathrm{mg} \mathrm{kg}^{-1}$ & 333 & $\pm \quad 1,5$ \\
\hline $\mathrm{Fe}, \mathrm{mg} \mathrm{kg}^{-1}$ & 25.294 & $\pm 377,4$ \\
\hline $\mathrm{Mn}, \mathrm{mg} \mathrm{kg}^{-1}$ & 300 & $\pm \quad 2,5$ \\
\hline $\mathrm{Cu}, \mathrm{mg} \mathrm{kg}^{-1}$ & 39 & 0,1 \\
\hline $\mathrm{Al}, \mathrm{mg} \mathrm{kg}^{-1}$ & 36.999 & $\pm 194,2$ \\
\hline
\end{tabular}

Laboratorio de Bromatología, INIA Remehue, Osorno, Chile. Datos calculados en base al peso seco, excepto el $\mathrm{N}$ total y $\mathrm{N}-\mathrm{NH}_{4}$ $\mathrm{n}=3 ; \pm$ error estándar de la media.

Norwalk, Connecticut, USA). El Ca, Mg, Zn, Fe, $\mathrm{Mn}, \mathrm{Cu}$ y Al fueron determinados por espectrofotometría de absorción atómica (Perkin-Elmer modelo 1100B, Norwalk, Connecticut, USA). El N total fue determinado por el método Kjeldahl (Gerhardt modelo Vapodest 5, Bonn, Alemania) de acuerdo a la metodología descrita por AOAC (1984). El N soluble (amonio) fue determinado por destilación directa y titulación (Gerhardt modelo Vapodest 12, Bonn, Alemania), según el método revisado por Sadzawka (1990).

Se establecieron cinco tratamientos: testigo absoluto ( $\sin$ fertilización), dosis de lodo baja $\left(50 \mathrm{t} \mathrm{ha}^{-1}\right)$, media $\left(100 \mathrm{tha}^{-1}\right)$ y alta $\left(200 \mathrm{tha}^{-1}\right)$ (equivalente a 95,190, y $380 \mathrm{~kg} \mathrm{~N}$ total ha ${ }^{-1}$ y 103,208, y $417 \mathrm{~kg}$ $\mathrm{P}_{2} \mathrm{O}_{5}$ ha $^{-1}$, respectivamente) y un tratamiento con fertilización inorgánica $\left(150 \mathrm{~kg} \mathrm{~N}\right.$ total $\mathrm{ha}^{-1} ; 350$ $\mathrm{kg} \mathrm{P}_{2} \mathrm{O}_{5}$ ha $^{-1}$ y $150 \mathrm{~kg} \mathrm{~K}_{2} \mathrm{O}$ ha $\left.^{-1}\right)$. Las dosis de lodo fueron estimadas de acuerdo al aporte de P. Se utilizaron parcelas de 4,5 × $3 \mathrm{~m}\left(13,5 \mathrm{~m}^{2}\right)$, en un diseño de bloques completos al azar con tres repeticiones. El suelo del sitio experimental se caracterizó químicamente al inicio en cada bloque y al final del experimento en cada tratamiento y repetición, en muestras obtenidas en el perfil de 0 a $20 \mathrm{~cm}$ de profundidad (Cuadro 2).

El cultivo de papa se estableció sobre una pradera utilizada en pastoreo con bovinos. La preparación del suelo se hizo en forma tradicional para el cultivo de papa, utilizando rastrajes, arado de discos y vibro cultivador. Previo a la plantación del ensayo de papa, el lodo se aplicó superficialmente y posteriormente se incorporó al suelo en los primeros 10 a $12 \mathrm{~cm}$ del perfil, mediante un rotovator (Gravely, modelo Professional, Brillion, Wiscosin, USA). El 6 de noviembre de 2002 se plantó papa cv. YaganaINIA, utilizando $2,5 \mathrm{t} \mathrm{ha}^{-1}$ de tubérculos calibre semilla, distanciadas a $75 \mathrm{~cm}$ entre hileras y $30 \mathrm{~cm}$ sobre la hilera.

El 28 de noviembre de 2002 se realizó el control de malezas con metribuzina en dosis de $0,48 \mathrm{~L}$ i.a. ha ${ }^{-1}$ (Sencor $480 \mathrm{SC}$, en dosis de $1,0 \mathrm{~L} \mathrm{ha}^{-1}$ de producto comercial, con concentración de 480 g i.a. $\mathrm{L}^{-1}$ ) y el control de hongos el 20 de febrero de 2003 con propamocarb $\left(0,94 \mathrm{~L}\right.$ i.a. ha $\left.{ }^{-1}\right)$ más clorotalomil $(0,94 \mathrm{~L}$ i.a. ha-1), utilizando Tatoo $\mathrm{C}$ en dosis de 2,5 $\mathrm{L} \mathrm{ha}^{-1} \mathrm{de}$ producto comercial, cuya concentración es de $375 \mathrm{~g}$ i.a. $\mathrm{L}^{-1}$ de cada componente, respectivamente).

Sesenta días después de la plantación se realizó la labor de aporca y luego de 117 días de desarrollo del cultivo (17 de marzo de 2003), se efectuó la cosecha en forma manual. Se evaluó el rendimiento total de papa $\left(\mathrm{t} \mathrm{ha}^{-1}\right)$, el peso por tubérculo $(\mathrm{g}) \mathrm{y}$ el número de tubérculos por planta. El rendimiento del cultivo se evaluó cosechando las dos hileras centrales, eliminando un metro lineal en los extremos de cada parcela. Los tubérculos se pesaron y separaron en los distintos calibres: consumo (sobre $65 \mathrm{~mm}$ ), semillas (entre 28 y $65 \mathrm{~mm}$ ) y bajo calibre (menor a $28 \mathrm{~mm}$ ).

Posterior a la cosecha de papa se realizó la siembra de ballica anual cv. Sabalan, mediante labor de cero labranza (21 de marzo de 2003), utilizando $35 \mathrm{~kg}$ $\mathrm{ha}^{-1}$. La ballica se evaluó en cuatro cortes con barra segadora (Gravely, modelo Professional, Brillion, Wiscosin, USA), en una superficie de $6 \mathrm{~m}^{2}$ por parcela. El material cosechado se pesó en verde y una submuestra de $200 \mathrm{~g}$ se deshidrató en un horno de ventilación forzada por 48 horas a $60{ }^{\circ} \mathrm{C}$ hasta peso constante, para determinar el contenido de materia seca (\% MS) y el rendimiento ( $\left.\mathrm{kg} \mathrm{MS} \mathrm{ha}{ }^{-1}\right)$. 


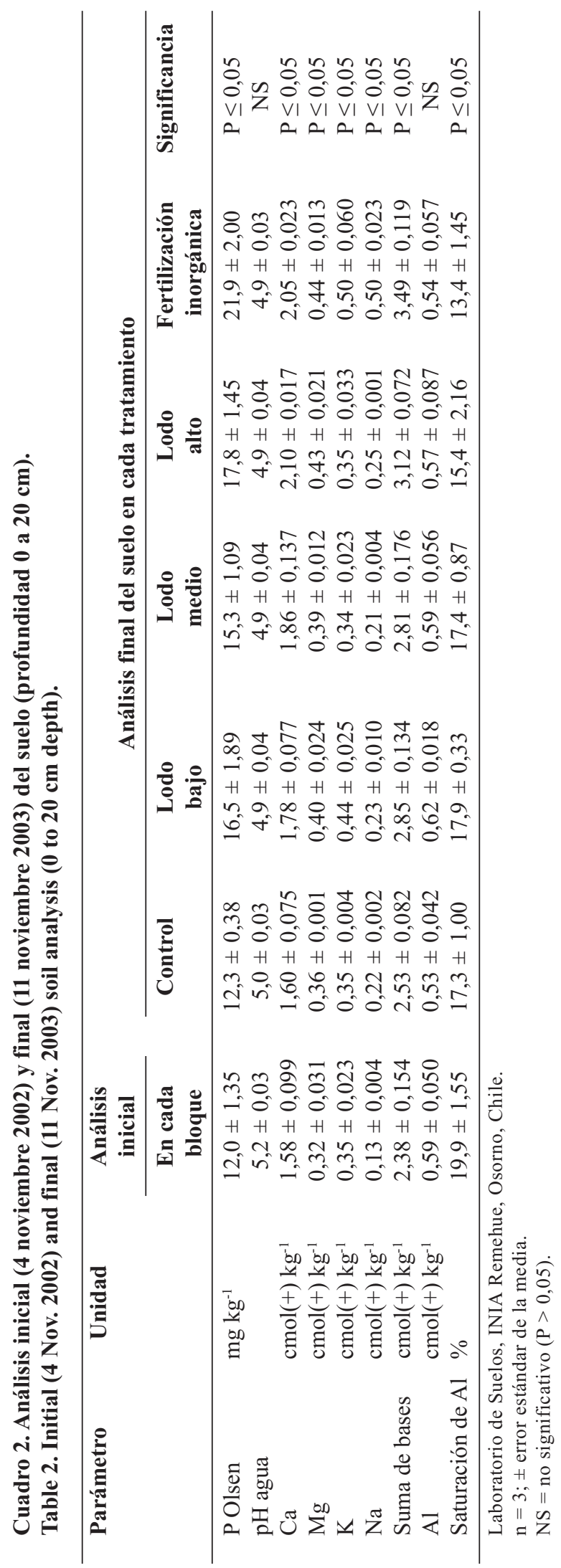

Los resultados se analizaron estadísticamente mediante análisis de varianza (ANDEVA) y prueba de diferencia mínima significativa (DMS al 5\%).

\section{RESULTADOS Y DISCUSIÓN}

\section{Análisis químico del lodo de lago}

El lodo de lago utilizado en el cultivo de papa presentó en su composición química una mezcla de macro y micro nutrientes necesarios para el crecimiento de plantas; sin embargo, en general sus contenidos fueron bajos (Cuadro 1). Estas concentraciones fueron más bajas que las reportadas para lodos de salmón y trucha colectados desde pisciculturas en tierra (Westerman et al., 1993; Naylor et al., 1999) y para purines de ganado y lodos de ciudad (MAFF, 2000), y similares a los lodos de salmón colectados bajo jaulas de crianza caracterizados por Salazar y Saldaña (2007).

El contenido de materia seca fue bajo, con una alta proporción de cenizas y una acidez moderadamente alcalina. El lodo tenía bajo contenido de materia orgánica, comparado con otros residuos orgánicos como los desechos de ciudad o purines de lechería, los que pueden contener sobre $80 \%$ de materia orgánica (Aguilera et al., 1995). El alto contenido de cenizas puede ser explicado por el sistema de extracción y por las condiciones ambientales donde se encuentra el lodo, pudiendo estar contaminado con arena del fondo lacustre.

El contenido de macro y micro nutrientes fue bajo, con un aporte de nitrógeno reducido, en especial la fracción soluble fácilmente asimilable por las plantas $\left(\mathrm{N}-\mathrm{NH}_{4}\right)$. El nutriente de mayor relevancia fue el fósforo $(\mathrm{P})$. En cuanto al contenido de micro nutrientes, si bien no había cantidades importantes tampoco se observaron valores que pudiesen ser restrictivos para el crecimiento y desarrollo del cultivo de papa.

Se destacó el elevado contenido de hierro $(\mathrm{Fe})$ y aluminio (Al), los cuales pueden ser explicados por la presencia de sedimentos del lago. Estos elementos son generalmente altos en los suelos volcánicos del sur de Chile (Sadzawka y Carrasco, 1985). El Al se encuentra principalmente en su forma no intercambiable (Salazar y Saldaña, 2007), por lo tanto produce bajo impacto negativo en el suelo.

El contenido de metales pesados no se analizó en los lodos utilizados para este experimento; sin em- 
bargo, resultados de la caracterización de lodos colectados bajo las mismas jaulas y otros sitios de crianza por Salazar y Saldaña (2007), muestran bajo contenido de metales pesados (cadmio, cromo, cobre, níquel, plomo y cinc), niveles que se encuentran por debajo del límite establecido por la Normativa Chilena (CONAMA, en prensa) y de la Comunidad Económica Europea (Council of the $\mathrm{Eu}-$ ropean Communities, 1986) para el uso de lodos domésticos y biosólidos en suelo agrícolas.

\section{Análisis químico del suelo}

El suelo del ensayo corresponde a un trumao típico de la zona sur de Chile, fuertemente ácido y alta saturación de aluminio (Cuadro 2). En general, en los aspectos nutricionales la fertilidad del suelo era moderada, con un contenido medio de P Olsen, pero con bajos niveles de cationes de intercambio, lo que puede estar asociado al historial de manejo del potrero, especialmente por la fertilización anterior. Destaca su alto porcentaje de saturación de aluminio, que limita la producción de los cultivos.

Los resultados del análisis de suelo posterior al cultivo de papa y previo a la siembra de la ballica anual (Cuadro 2), indicaron que los parámetros químicos del suelo como P Olsen, $\mathrm{Ca}, \mathrm{Mg}, \mathrm{K}, \mathrm{Na}$, la suma de bases y la saturación de Al, fueron afectados significativamente $(\mathrm{P} \leq 0,05)$ por la adición de distintas dosis de lodo. Sin embargo, cabe destacar que los mayores efectos observados en las propiedades químicas del suelo se obtuvo en el tratamiento donde se aplicó la fertilización inorgánica, el que además de los parámetros anteriormente indicados, mostró diferencias significativas $(\mathrm{P} \leq$ $0,05)$ en el contenido de $\mathrm{NO}_{3}^{-}$, al compararlo con el tratamiento control y en algunos parámetros con los tratamientos que incluyeron lodo (antecedentes no mostrados). En el análisis final del suelo no se observaron diferencias estadísticas $(\mathrm{P}>0,05)$ en el $\mathrm{pH}$ al agua, el contenido de $\mathrm{NH}_{4}^{+}$(dato no mostrado) y Al disponible, por la incorporación de las diferentes dosis de lodo al suelo.

\section{Desarrollo del cultivo de papa}

Independiente de la dosis incorporada al suelo, el lodo de salmones producidos en el lago no afectó la emergencia de los brotes ni el desarrollo vegetativo posterior del cultivo de papa. Esta apreciación visual en el cultivo de papa concuerda con los resultados obtenidos por Bustos et al. (2003) en ballica anual establecida en siembra directa, trabajo en que se incorporaron distintas dosis de lodo proveniente de la crianza de salmones en agua dulce (lago) y en agua salada (mar). Como antecedente complementario se señala que no se observaron efectos adversos en la capacidad de brotación de los tubérculos de papa. Tampoco se afectó el desarrollo morfológico de los cultivos evaluados en este trabajo.

La incorporación al suelo de dosis incrementales de lodo de salmones no mostró diferencias significativas en el rendimiento total de tubérculos $(\mathrm{P}>$ $0,05)$ entre los tratamientos con lodo. Sin embargo, el rendimiento total fue significativamente mayor en el tratamiento con la fertilización inorgánica (Cuadro 3), con respecto a cualquiera de las dosis de lodo.

La baja respuesta del cultivo de papa al uso de dosis crecientes de lodo se podría explicar por el escaso aporte de nitrógeno rápidamente disponible de este residuo orgánico, siendo la mayor parte constituido por $\mathrm{N}$ orgánico de baja o lenta liberación (Mazzarino et al., 1997; Pinochet et al., 2001).

No se obtuvo diferencias significativas $(\mathrm{P}>0,05)$ en el peso promedio de tubérculos entre los tratamientos evaluados, pero el número de tubérculos por planta fue significativamente superior $(\mathrm{P} \leq 0,05)$ en el tratamiento con fertilización inorgánica.

La proporción de los distintos calibres de tubérculos fue similar entre los tratamientos evaluados (Figura 1). La proporción del calibre semillas varió entre 74 y $83 \%$ del rendimiento total de tubérculos para todos los tratamientos, mientras que el calibre consumo alcanzó un máximo de $20 \%$ del rendimiento total en la dosis alta de lodo. La categoría de bajo calibre no superó el 7\% del rendimiento total de papa, independiente de los tratamientos (Figura 1). La alta proporción de tubérculos en el calibre semillas podría explicarse en parte por el atraso en la fecha de plantación (6 de noviembre de 2002) con respecto al período recomendable para la Región de Los Lagos, el que se extiende entre fines de septiembre a finales de octubre (Peralta, 2006).

Al considerar los resultados obtenidos con las diferentes dosis de lodo de salmones incorporadas al suelo en el cultivo de papa y su nulo daño a la vegetación, es posible pensar en la alternativa de aplicar lodo en combinación con una mezcla de fertili- 
Cuadro 3. Efecto de los distintos tratamientos en el rendimiento total ( $\left(\mathrm{ha}^{-1}\right)$, peso por tubérculo y número de tubérculos por planta, en papa cv. Yagana-INIA.

Table 3. Effect of different treatments on total yield $\left(\mathrm{t} \mathrm{ha}^{-1}\right)$, weight per tuber and number of tubers per plant in potato cv. Yagana-INIA.

\begin{tabular}{lccr}
\hline Tratamientos & $\begin{array}{c}\text { Rendimiento } \\
\left(\mathbf{t} \mathbf{~ h a}^{-1}\right)\end{array}$ & $\begin{array}{c}\text { Peso por } \\
\text { tubérculo }(\mathbf{g})\end{array}$ & $\begin{array}{c}\text { Tubérculos por } \\
\text { planta }\left(\mathbf{N}^{\mathbf{}}\right)\end{array}$ \\
\hline Control & $39,4 \pm 2,76 \mathrm{~b}$ & $81,8 \pm 6,53$ & $9,8 \pm 0,97 \mathrm{~b}$ \\
Lodo bajo & $47,1 \pm 3,25 \mathrm{~b}$ & $88,8 \pm 11,09$ & $10,2 \pm 1,45 \mathrm{~b}$ \\
Lodo medio & $45,6 \pm 0,87 \mathrm{~b}$ & $76,5 \pm 13,72$ & $10,4 \pm 0,34 \mathrm{~b}$ \\
Lodo alto & $47,5 \pm 8,83 \mathrm{~b}$ & $93,1 \pm 9,53$ & $11,5 \pm 0,49 \mathrm{~b}$ \\
Fertilización inorgánica & $64,3 \pm 7,53 \mathrm{a}$ & $76,8 \pm 11,67$ & $16,2 \pm 0,22 \mathrm{a}$ \\
DMS 5\% & 13,57 & $\mathrm{NS}$ & 2,25 \\
\hline
\end{tabular}

Valores promedio dentro de una columna seguidos por diferente letra difieren estadísticamente según prueba DMS (P $\leq 0,05)$. $\mathrm{n}=3 ; \pm$ error estándar de la media; NS: no significativo.

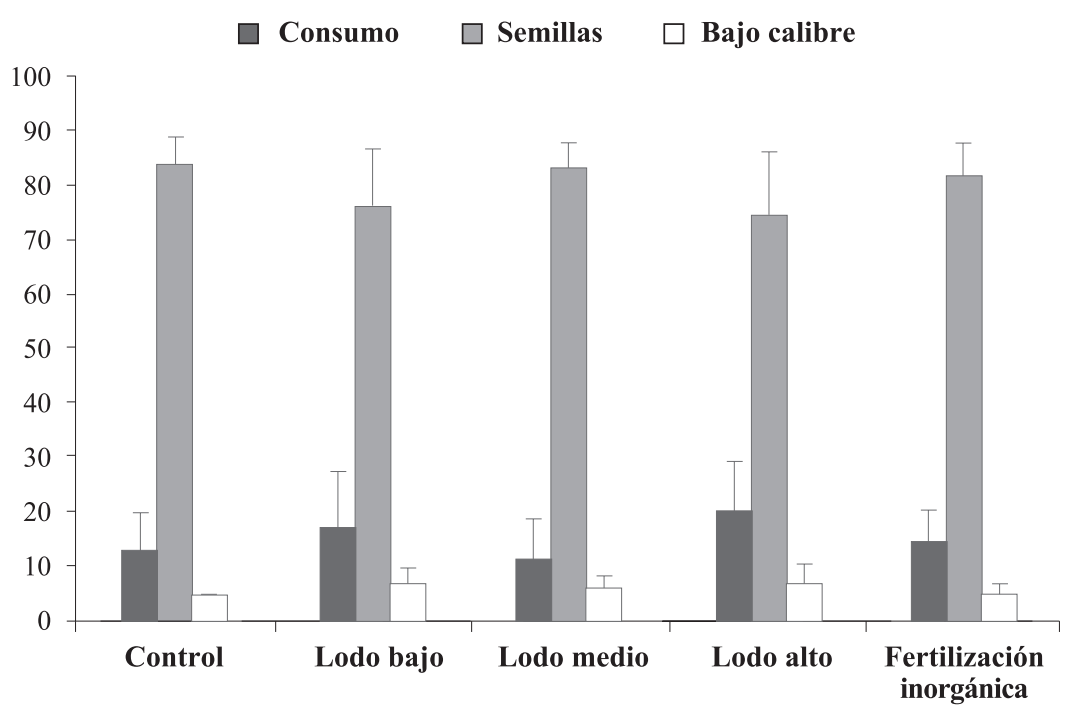

Figura 1. Distribución porcentual de los distintos calibres en papa cv. Yagana-INIA obtenidos en los tratamientos control, diferentes dosis de lodo de salmones y fertilización inorgánica.

Figure 1. Distribution (\%) of different calibres of potato $\mathrm{cv}$. Yagana-INIA obtained with control, increasing salmon sludge doses and inorganic fertilizer treatment.

zantes inorgánicos como nutrición base para el cultivo. Sin embargo, es necesario considerar el costo del transporte entre el sitio de origen y el predio, especialmente por el bajo contenido de materia seca del producto (Cuadro 1), además del costo de aplicación e incorporación al suelo.

A pesar de lo anterior, el reciclaje de estos desechos orgánicos en suelos agrícolas es una alternativa factible; sin embargo, antes de masificar su uso en sistemas agrícolas se requieren mayores estu- dios relacionados a la complementación con fertilizantes inorgánicos y evaluar el potencial incremento de metales pesados en el suelo y/o otros productos químicos utilizados en la crianza de salmonídeos (e.g., antibióticos) en especial el posible efecto acumulativo al utilizar altas dosis de lodo en años sucesivos en un mismo suelo.

\section{Rendimiento de la ballica anual}

El efecto residual del lodo en el rendimiento de materia seca (MS) de la ballica anual fue significa- 
tivamente diferente entre el control y los otros tratamientos (Cuadro 4). Los tratamientos con lodo bajo y alto produjeron entre 28 y $38 \%$ más MS que el control, mientras que el tratamiento con fertilización inorgánica rindió un $54 \%$ más que el testigo (5.025 kg MS ha-1), al considerar los cuatro cortes efectuados entre la siembra realizada en marzo y la cosecha de la ballica, simulando el corte para conservación como ensilaje en noviembre. Estos resultados son concordantes con los obtenidos por Teuber et al. (2005), quienes utilizaron lodo de salmones criados en el mar.

El bajo efecto residual en el rendimiento de materia seca de las diferentes dosis de lodo incorporado, se explica por la lenta tasa de mineralización del nitrógeno contenido en este desecho orgánico (Mazzarino et al., 1997; Pinochet et al., 2001). En un estudio de mineralización realizado con lodos de trucha, Westerman et al. (1993) mostraron un

Cuadro 4. Efecto residual del lodo de salmón y de la fertilización inorgánica en el rendimiento de la ballica anual cv. Sabalan.

Table 4. Residual effect of salmon sludge and inorganic fertilizer on the annual ryegrass cv. Sabalan yield.

\begin{tabular}{lc}
\hline Tratamientos & $\begin{array}{c}\text { Rendimiento acumulado } \\
\left(\mathbf{k g ~ M S ~ h a} \mathbf{~}^{-1}\right)\end{array}$ \\
\hline Control & $5.025 \pm 899,7 \mathrm{c}$ \\
Lodo bajo & $6.424 \pm 452,8 \mathrm{~b}$ \\
Lodo medio & $6.465 \pm 237,7 \mathrm{~b}$ \\
Lodo alto & $6.925 \pm 116,0 \mathrm{ab}$ \\
Fertilización inorgánica & $7.722 \pm 116,0 \mathrm{a}$ \\
DMS 5\% & $1.264,5$ \\
\hline
\end{tabular}

Valores dentro de una columna seguidos por igual letra, no difieren estadísticamente según prueba DMS (Diferencia mínima significativa, $\mathrm{P} \leq 0,05) . \mathrm{n}=4, \pm$ error estándar de la media. potencial de $\mathrm{N}$ disponible de $20 \%$ para el cultivo. Por su parte Mazzarino et al. (1998) determinaron valores entre 10 y $50 \%$ de mineralización de $\mathrm{N}$ para lodos colectado bajo jaulas de salmones. Alfaro et al. (2004) determinaron un 39\% de mineralización de $\mathrm{N}$ en un período de 90 días en experimentos realizados en macetas bajo condiciones ambientales controladas, evaluando un lodo similar al utilizado en el presente estudio.

\section{CONCLUSIONES}

La incorporación de lodo proveniente de la crianza intensiva de salmonídeos en lago no aumentó el rendimiento del cultivo de papa en comparación con el tratamiento con fertilización inorgánica. No hubo diferencias significativas de rendimiento de los tratamientos con lodo y el control sin fertilización.

Hubo efecto residual del nitrógeno en el rendimiento de materia seca de la ballica anual sembrada inmediatamente después de la cosecha de papa. Los rendimientos de MS obtenidos fueron más altos en los tratamientos con lodo que en el control sin fertilización, siendo similares el rendimiento en la dosis de lodo alta y la fertilización inorgánica.

En el suelo se observó un aumento en el fósforo disponible en los tratamientos con dosis de lodo alto y la fertilización inorgánica. Con la dosis alta de lodo también hubo aumento en la suma de bases y reducción en el porcentaje de saturación de aluminio, mejorando la calidad química del suelo.

\section{RECONOCIMIENTO}

Investigación financiada por el Proyecto FONDEF D01I11-13.

\section{LITERATURA CITADA}

Ackefors, H., and M. Enell. 1994. The release of nutrients and organic matter from aquaculture systems in Nordic countries. Journal of Applied Ichthyology-Zeitschrift Fur Angewandte Ichthyologie 10:225-241.

Aguilera, S., G. Borie, P. Peirano, M. Mora, y R. Demanet. 1995. Caracterización de purines para su potencial uso como fertilizante y mejorador de suelos. Agric. Téc. (Chile) 55:251-256.
Alfaro, M.A., F.J. Salazar, y A. Valdebenito. 2004. Nitrogen mineralization from fish sludge as affected by plant uptake. p. 136-137. In Hatch, D., D.R. Chadwick, S. Jarvis, and A. Roker (eds.). Proceedings 12th Nitrogen Workshop: Controlling N Flows and Losses. Exeter. 21 al 24 de Septiembre del 2004. Institute of Grassland and Environmental Research, Exeter, Devon, United Kingdom. 
AOAC. 1984. Official methods of analysis. 1141p. $14^{\text {th }}$ ed. Association of Official Agricultural Chemist (AOAC), Washington, DC, USA.

Bustos, C., N. Teuber, F. Salazar, D. Pinochet, y A. Valdebenito. 2003. Efecto de diferente dosis y origen de lodo de salmón en el establecimiento de ballica anual. p. 35-36. In XXVIII Reunión Anual Sociedad Chilena de Producción Animal. Talca. 15-17 de octubre de 2003. Universidad Católica del Maule, Talca, Chile.

CIREN. 2003. Descripciones de suelos, materiales y símbolos. Estudio agrológico X Región. Tomo II. 412 p. Publicación $\mathrm{N}^{\circ}$ 123. Centro de Información de Recursos Naturales (CIREN), Santiago, Chile.

CONAMA. 2007. Borrador del Proyecto definitivo de reglamento sobre manejo de lodos no peligrosos. 22 p. Comisión Nacional del Medio Ambiente (CONAMA), Santiago, Chile. (En prensa).

Council of the European Communities. 1986. Council Directive on the protection of the environment, and in particular of the soil, when sewage sludge is used in agriculture 86/278/EEC. Official Journal of the Europen Communities, 4.7.86. $\mathrm{N}^{\circ}$ L181 p. 6-12.

Laos, F., P. Satti, I. Walter, M.J. Mazzarino, and S. Moyano. 2000. Nutrient availability of composted and noncomposted residues in a Patagonian Xeric Mollisol. Biol. Fertil. Soils 31:462-469

MAFF. 2000. Fertiliser recommendations for agricultural and horticultural crops. 176 p. Reference Book 209. $7^{\text {th }}$ ed. HMSO, Ministry of Agriculture, Fisheries and Food (MAFF), London, United Kingdom.

Mazzarino, M.J., F. Laos, P. Satti and S. Moyano. 1998. Agronomic and environmental aspects of utilization of organic residues in soils of the Andean Patagonian region. Soil Sci. Plant Nutr. 44:105-113.

Mazzarino, M.J., I. Walter, G. Costa, F. Laos, L. Roselli, and P. Satti. 1997. Plant response to fish farming wastes in volcanic soils. J. Environ. Qual. 26:522-528.

Naylor, S., R. Moccia, and G. Durant. 1999. The chemical composition of settleable solid fish waste (manure) from commercial Rainbow trout farm in Ontario, Canada. N. Am. J. Aquaculture 61:21-26.

Niklitschek, E., D. Soto, and A. Lafon. 2006. Trade liberalization, rural poverty and the environment. A case study of forest and salmon sectors in Chile. The Salmon Sector. 25 p. World Wildlife Fund-World Bank Report CT 05-02. Santiago, Chile.

Peralta, J. 2006. Estrategias de riego en papa para la zona sur de Chile. p. 101-114. In Rojas, J. y S. Orena (eds.). Manual de producción de papa para la agricultura familiar campesina (Convenio INIA-INDAP). Boletín INIA $N^{\circ}$ 147. Instituto de Investigaciones Agropecuarias, Centro Regional de Investigación Remehue, Osorno, Chile.
Pinochet, D., P. Artacho, y P. Azúa. 2001. Potencialidad como abono orgánico de los desechos sólidos subproductos de especies salmonídeas. Agro Sur 29:78-82.

Rodríguez, R, 1993. Determinación cuantitativa de la carga de desechos ingresados al lago Ranco por efecto del cultivo de especies salmonídeas. $156 \mathrm{p}$. Tesis de grado Licenciado en Biología Marina. Facultad de Ciencias, Universidad Austral de Chile, Valdivia (Chile).

Sadzawka, A., 1990. Métodos de análisis de suelos. INIA serie La Platina $\mathrm{N}^{\circ} 16.127$ p. Instituto de Investigaciones Agropecuarias, Ministerio de Agricultura, Santiago, Chile.

Sadzawka, A. y M. Carrasco. 1985. Química de los suelos volcánicos. p. 337-490. In Tosso, J. (ed.). Suelos volcánicos de Chile. Instituto de Investigaciones Agropecuarias, Santiago, Chile.

Salazar, F.J., and R.C. Saldaña. 2007. Characterization of manures from fish cage farming in Chile. Bioresour. Technol. 98:3322-3327.

Salmón Chile. 2006. Salmonoticias y estadísticas productivas. Disponible en http://www.salmonchile.cl/ Leído marzo 2007.

Smith, J.H. 1985. Fertilizing agricultural land with Rainbow trout manure for growing silage corn. Soil Sci. Soc. Am. J. 49:131-134.

Stickney, R.R. 2002. Impacts of cage and net pen culture on water quality and benthic communities. p. 105118. In Tomasso, J. (ed.). Aquaculture and the environment in the United States. U.S. Aquaculture Society, Baton Rouge, Louisiana, USA.

Teuber, N., M.A. Alfaro, F.J. Salazar, and C. Bustos. 2005. Sea salmon sludge as fertilizer: effects on a volcanic soil and annual ryegrass yield and quality. Soil Use Managem. 21:432-434.

Teuber, N., F. Salazar, y A. Valdebenito. 2004. Respuesta del cultivo de papa y efecto residual en ballica anual a la incorporación de diferentes dosis de lodo proveniente de la producción intensiva de salmonídeos. p. 207-215. Boletín No 20. Simposio Residuos Orgánicos y su Uso en Sistemas Agroforestales. Sociedad Chilena de la Ciencia del Suelo, Universidad de La Frontera, Temuco, Chile.

Westerman, P.W., J.M. Hinshaw, and J.C. Barker. 1993. Trout manure characterization and nitrogen mineralization rate. p. 35-43. In Wang, J.K (ed.). Techniques for modern aquaculture. American Society of Agricultural Engineers, St. Joseph, Michigan, USA. 